\title{
Impatience with Inpatients: Are Hospitalization Rates Declining for IBD Patients?
}

\author{
Anand Kumar ${ }^{1,2} \cdot$ Arun Swaminath ${ }^{1,2}$ \\ Accepted: 24 August 2021 / Published online: 16 September 2021 \\ (c) The Author(s), under exclusive licence to Springer Science+Business Media, LLC, part of Springer Nature 2021
}

The annual costs of inflammatory bowel disease (IBD) care exceed \$14 billion in the United States alone, of which hospitalization-related expenditure accounts for nearly two thirds of the cost [1]. The rising incidence and prevalence of IBD places an even greater stress on health care systems. Evaluation of current and past IBD hospitalization and readmission trends will help develop policies necessary to address the challenges faced by health systems in providing high-quality and cost-effective care for this patient population.

Historically, the lack of safe and effective maintenance therapy led to unplanned hospitalizations for disease flares and complications, significantly contributing to the healthcare burden and costs associated with IBD. Nevertheless, in the last two decades there have been several changes to the management of patients with IBD including increased use of biologic therapies, and adoption of more objective treatment targets including steroid-free clinical response, biomarker targets, and endoscopic healing [2]. These practice changes were aimed at achieving superior disease control and shifting the management of IBD from high-cost inpatient hospital care to lower-cost close monitoring in ambulatory care settings [3]. Despite these advancements, it remains unclear if the risk of unplanned IBD-related hospitalizations, a useful surrogate for improved disease control, has been reduced.

The existing data on the risk of hospitalization and its trend over time among patients with IBD are exceedingly heterogenous. Hospital-based studies are prone to selection bias and not representative of the entire IBD population in the community. National databases based on discharge codes or insurance claims, although useful to assess the disease

Anand Kumar

kirar.anand@gmail.com

1 Division of Gastroenterology, Lenox Hill Hospital, New York, NY, USA

2 Donald and Barbara Zucker School of Medicine at Hofstra/Northwell, Hempstead, NY, USA burden within a region, do not provide patient-level risk estimates necessary for risk stratification and development of risk-based treatment algorithms. Therefore, the most accurate method to estimate the risk of hospitalization among IBD patients is by longitudinally tracking all patients with IBD in a community from the time of their diagnosis up to their death. Yet, such population-based inception cohort data are limited as they are often challenging to maintain and regulated by governmental policies and healthcare delivery systems.

The study by Tsai et al. published in this issue of Digestive Diseases and Sciences is a systematic review and metaanalysis of population-based inception cohort studies (published up to September 3, 2019) designed to estimate the cumulative risk of IBD-related and all-cause hospitalization in patients with ulcerative colitis (UC) and Crohn's disease (CD) [4]. They included 6 cohorts of patients with UC (17,190 patients) finding the cumulative 1-, 3- and 5-year risk of UC-related hospitalization to be $10.4 \%$ (95\% CI, 8.2-13.2), $17.0 \%$ (95\% CI, 14.0-20.4) and $21.5 \%$ (95\% CI, 18.0-25.4), respectively. In the 6 cohorts of patients with CD (23,081 patients), the cumulative 1-, 3-and 5-year risk of CD-related hospitalization was $29.3 \%$ (95\% CI, 20.0-40.8), $38.5 \%$ (95\% CI, 26.8-51.7) and 44.3\% (95\% CI, 32.7-56.5), respectively. This implies nearly a 2 - to 3 -fold risk of disease-related hospitalization with $\mathrm{CD}$ as compared with UC patients at each of the three follow-up intervals. In general, the natural history of $\mathrm{CD}$ carries a higher disease burden compared with UC, since untreated CD is associated with complications from progressive bowel damage such as strictures, fistulae and abscesses that often require hospitalization for medical and surgical treatments. Prior studies have reported a similar trend of higher hospitalization rates among CD cohorts compared with patients with UC $[5,6]$.

On meta-regression analysis, the study found younger age at onset was associated with increased risk of hospitalization in UC and ileal-dominant and perianal disease was associated with increased risk of hospitalization in CD [4]. 
Nonetheless, as the number of studies analyzed was $<10$, the authors cautioned that the meta-regression may be underpowered, with the caveat that these results should be interpreted cautiously. Furthermore, based on qualitative assessment of risk factors, the authors reported that in UC, extensive colitis and early need for corticosteroids and/ or immunomodulators were consistently associated with higher hospitalization risk [7]. Whereas, penetrating and/or fistulizing disease and early need for corticosteroids, immunomodulators and/or tumor necrosis factor antagonists were associated with increased risk CD-related hospitalization in majority of included studies [5]. It is noteworthy that, previously, immunomodulators and biologic therapies were often reserved for the sickest stratum of IBD patients and therefore their early use in the disease course was likely a surrogate for aggressive disease behavior rather than treatment failure. However, now with the changing practice of early intensive therapy in IBD, further studies will be needed to evaluate if that would help reduce the risk of hospitalizations.

In contrast to initial hospitalization, the study found that the risk of readmission was higher in patients with UC relative to those with CD. Though this finding is consistent with some prior studies [8], the underlying reason is unclear, and was hypothesized to be related to the typical flare symptoms in UC (i.e., urgency and rectal bleeding) that may prompt patients to seek urgent treatment, compared with the chronic/ indolent abdominal pain that may predominate in patients with $\mathrm{CD}$.

Another important aspect of this meta-analysis was the observed steady decline in risk of hospitalization in patients diagnosed in a more recent era, both on meta-regression (based on mid-point of cohort entry) and on sensitivity analysis of IBD patients diagnosed after year 2000. Authors hypothesized that this decline in hospitalization trend could be a result of improved disease outcomes related to early diagnosis, access to gastroenterologists specializing in IBD, early use of disease-modifying immunosuppressive therapy, and proactive disease monitoring. Nevertheless, it is noteworthy that individual studies differed with regard to hospitalization trends in the contemporary era of biologic therapies. An analysis of 369 patients with UC from Olmsted County, MN, found a reduction in incidence rate of hospitalization over the last four decades, although cumulative probability of first hospitalization increased with successive decades of diagnosis [7]. Jeuring et al. found that compared to a past reference period of 1991-1998, between 2006 and 2011, the hospitalization rates decreased in patients with $\mathrm{CD}$; interestingly, progression to complicated disease was still common and immunomodulator users had a similar risk of hospitalization, surgery, or phenotype progression as matched nonusers, implying that the reduction in hospitalization was related to other factors such as improved disease monitoring [9]. A study utilizing the Dutch population-based
IBD cohort reported non-statistically significant numerical differences in the 5-year cumulative UC-related hospitalization risk among patients diagnosed in three successive time periods i.e., 1991-1997, 1998-2005 and 2006-2010 (22.3 vs 19.5 vs $18.3 \%$, respectively, $p=0.10$ ) despite increased use of immunomodulators and biologics over these years [10]. A study of the Epi-IBD cohort - a population-based inception cohort of patients with IBD diagnosed in 2010 in 22 European countries and Israel - found that immunomodulator (but not biologic) use was associated with reduced hospitalization risk in UC [11]. Another study reported no significant change in hospitalization trends before and after 2010 despite higher exposure to anti-tumor necrosis factor in IBD in the Québec provincial database [6]. These studies suffer from significant heterogeneity; not only do they originate in different countries with differences in patient characteristics and healthcare practices, but also from different time points of IBD diagnosis. There were also differences in the timing of adoption of biologic therapy among countries. For example, infliximab was approved by the US FDA for use in IBD in 1998, whereas the same was approved for use in by Health Canada for IBD in 2005-2006. Newer biologics and small molecules including vedolizumab, ustekinumab, and tofacitinib have only become available after 2014 . There could be differences in thresholds for hospitalization such as hospitalizations for biologic infusion could be coded as IBDrelated even if not a true flare or complication. In which case one needs to focus on the cumulative length of stay (rather than frequency of hospitalization) which has decreased over the years based on few studies [9]. Interestingly, studies comparing other outcomes such as healthcare cost and death rates prior to and after introduction of biologics were also unable to demonstrate a definitive improvement in those aspects [12]. Therefore, despite the observed decline in IBD-related hospitalization trend in this meta-analysis, the results are subject to multiple possible confounders. Moreover, it remains unclear if these results reflect the effects of increased adoption of immunotherapy and stricter treatment guidelines.

As correctly noted by the authors, their meta-analysis suffers from considerable heterogeneity in most analyzed outcomes. Meta-regression was also limited by the small number of included studies. The study was unable to examine factors that may have contributed to a decline in risk of hospitalization. Further heterogeneity may have been introduced by combining risk of IBD-related hospitalization with those that did not specify the reason for hospitalization and those that only reported all-cause hospitalization assuming that IBD-flare was the likely cause of hospitalization in these cohorts.

In summary, the present study systematically analyzed 10 population-based inception IBD cohorts in order to provide a pooled estimate of cumulative incidence of IBD-related and 
all-cause hospitalization in patients with UC and CD. The study reported a decline in hospitalization risk in patients diagnosed in the last two decades compared with those diagnosed earlier. Further real-world studies analyzing outcomes in patients with IBD diagnosed in the current era will expand understanding of the potential impact of new therapies and specific treatment strategies aimed at improving the quality of care in IBD patients.

\section{References}

1. Mehta F. Report: economic implications of inflammatory bowel disease and its management. Am J Manag Care. 2016;22:s51-60.

2. Turner D, Ricciuto A, Lewis A et al. STRIDE-II: An update on the selecting therapeutic targets in inflammatory bowel disease (STRIDE) initiative of the international organization for the study of IBD (IOIBD): determining therapeutic goals for treat-to-target strategies in IBD. Gastroenterology. 2021;160:1570-1583.

3. Colombel JF, Narula N, Peyrin-Biroulet L. Management strategies to improve outcomes of patients with inflammatory bowel diseases. Gastroenterology 2017;152:351-361e55.

4. Tsai L, Nguyen NH, Ma C, Prokop LJ, Sandborn WJ, Sing S. Systematic Review and Meta-analysis: Risk of Hospitalization in Patients with Ulcerative Colitis and Crohn's Disease in Population-based Cohort Studies. Dig Dis Sci. (Epub ahead of print). https://doi.org/10.1007/s10620-021-07200-1.

5. Niewiadomski O, Studd C, Hair C et al. Prospective populationbased cohort of inflammatory bowel disease in the biologics era: Disease course and predictors of severity. J Gastroenterol Hepatol 2015;30:1346-1353.
6. Verdon C, Reinglas J, Coulombe J et al. No change in surgical and hospitalization trends despite higher exposure to anti-tumor necrosis factor in inflammatory bowel disease in the québec provincial database from 1996 to 2015. Inflamm Bowel Dis 2021;27:655-661.

7. Samuel S, Ingle SB, Dhillon S et al. Cumulative incidence and risk factors for hospitalization and surgery in a population-based cohort of ulcerative colitis. Inflamm Bowel Dis 2013;19:1858-1866.

8. Gajendran M, Umapathy C, Loganathan P, Hashash JG, Koutroubakis IE, Binion DG. Analysis of hospital-based emergency department visits for inflammatory bowel disease in the USA. Dig Dis Sci 2016;61:389-399.

9. Jeuring SF, van den Heuvel TR, Liu LY et al. Improvements in the long-term outcome of Crohn's disease over the past two decades and the relation to changes in medical management: results from the population-based IBDSL Cohort. Am J Gastroenterol 2017;112:325-336.

10. Jeuring SF, Bours PH, Zeegers MP et al. Disease outcome of ulcerative colitis in an era of changing treatment strategies: results from the Dutch population-based IBDSL Cohort. J Crohns Colitis. 2015;9:837-845.

11. Burisch J, Katsanos KH, Christodoulou DK et al. Natural disease course of ulcerative colitis during the first five years of follow-up in a European population-based inception Cohort-An Epi-IBD study. J Crohns Colitis. 2019;13:198-208.

12. Nocerino A, Feathers A, Ivanina E, Durbin L, Swaminath A. Mortality risk of inflammatory bowel disease: a case-control study of New York state death records. Dig Dis Sci 2019;64:1604-1611.

Publisher's Note Springer Nature remains neutral with regard to jurisdictional claims in published maps and institutional affiliations. 